\section{Compliance with first time spectacle wear in children under eight years of age}

\section{Abstract}

An anonymous prospective survey was carried out of children under 8 years of age prescribed a first pair of spectacles from the Royal Berkshire Hospital between August 1995 and May 1996, focusing on compliance in relation to refractive error, visual status and social factors. One hundred and thirty-three children were surveyed. Mean compliance was high (79.5/100) and spectacles were well liked by most children. Improvement in vision had little or no relationship with compliance. Significant factors were fit and what friends said about the spectacles. Adverse comments were rare, especially in the younger children, but increased with age. In pre-school children spectacles did not appear to be the salient feature in negative social judgements that has been found to occur in adults.

Key words Compliance, Spectacles, Pre-school children, Social salience, Screening

Few medical treatments for children are as long term and as visible to others as spectacles. The key to their effectiveness in the treatment of amblyopia and strabismus is that they are worn constantly. Poor or reluctant compliance results either in the spectacles not being worn, or their being the cause of stress for the child and its family as wear is enforced. Published literature is sparse, ${ }^{1}$ but one study ${ }^{2}$ suggests that spectacles are not salient to facial recognition or social judgements in young children, suggesting that younger children are less likely to be influenced by the adverse stereotypes that occur in adults, and so have fewer problems in accepting and being accepted in spectacles.
The present study was carried out to find out exactly how well young children settle into a new pair of spectacles and to determine whether age, visual improvement or social factors influence good compliance.

\section{Method}

The study involved every child prescribed a first pair of spectacles from the Eye Department of the Royal Berkshire Hospital between August 1995 and May 1996 and looked at the first few weeks of wear. This initial period of wear was chosen because from clinical experience it appears crucial to moulding a habit of wear, and also, in a frequently amblyopic population, compliance with spectacles at this time was not confused with compliance with the occlusion often prescribed after a few weeks of spectacles alone.

When the child returned for the 6-week follow-up visit the parents were asked to participate in the study, a full explanation was given and they were handed a questionnaire to complete at home and return by post. The orthoptist also completed a questionnaire. The two questionnaires were paired and numbered, but not otherwise identifiable, and were matched up as the parental form was returned. Efforts were made to ensure that the parents were aware that their responses could not be personally identified and that even if compliance was poor their responses would be anonymous.

Questionnaire items were selected after studying literature on compliance in many areas of medicine and after consultation with psychologists at the University of Reading (Tables 1 and 2).

Where an interval scale was available, such as age or visual acuity, this was used. Where a scientific scale was not available most responses were required to be marked along a visual analogue scale (ranging from maximum/best possible to minimum/worst possible) and were
A.M. Horwood Orthoptic Department Royal Berkshire Hospital London Road, Reading Berkshire RG1 5AN, UK Tel: $+44(0) 1189877683$ 
Table 1. Orthoptist questionnaire items

Age

Prescription

VA at visits before and after spectacles (converted to logMAR equivalent)

Any binocular benefit (e.g. better stereoacuity or control of deviation)? Y / N

Would both-eyes-open VA have improved (e.g. in fixing eye of amblyope)? Y/N

Estimate of spectacles fit (VAS)

Proportion of time worn (VAS)

General impression of compliance based on whole clinic visit (VAS)*

How much child apparently likes the spectacles (VAS)

Answers to the following questions asked by the orthoptist:

'Do you think you can see better with your glasses?' $\mathrm{Y} / \mathrm{N}$

'What do your friends say about you in your glasses?' (comment recorded)

'What do the people you don't like say about you in your glasses' (comment recorded)

VA, visual acuity; VAS, visual analogue scale.

*Main compliance measure.

later scored from zero to 100 . Such scales are commonly used in psychological and social science research to quantify attitude and social data and may be analysed (although with some caution) using parametric statistical tests. $^{3,4}$

Although different tests were used, visual acuities were converted to logMAR units for analysis and the same test was always used for each child.

\section{Results}

One hundred and thirty-eight children were eligible for inclusion. Most had had their spectacles for about

5 weeks, depending on the speed of obtaining them from the optician. Four were excluded for reasons not relevant to the study and one parent declined to take part, so 133 children formed the main study group. One hundred and four $(78 \%)$ complete sets of data were obtained. Twentysix $(19.5 \%)$ parents failed to return the questionnaire (a

Table 2. Parental questionnaire items

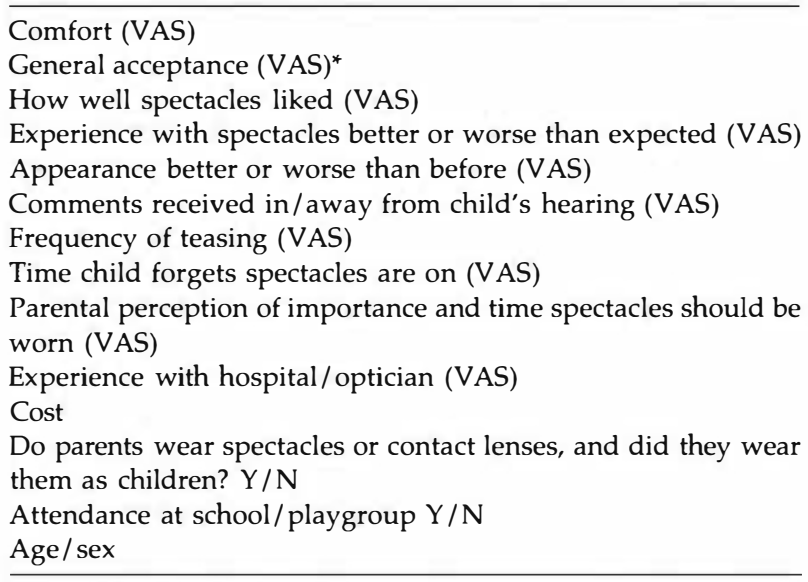

VAS, visual analogue scale.

*Main parental compliance measure. percentage that compares favourably with other postal surveys). Analysis of data from the children for whom forms were not returned showed no significant difference in the orthoptist's estimate of compliance (although the non-returners had a slightly better mean score), prescription, vision or diagnosis relative to children for whom forms had been returned, suggesting that reasons associated with poor compliance were not the reason for dropout. This, the efforts made to guarantee and explain anonymity of the replies, and the high correlation between the orthoptist's and parents' estimates of compliance (see later) made it seem reasonable to include data from the orthoptist questionnaires in the analysis, where appropriate, even if the parental forms were missing.

Age distribution of subjects was according to Fig. 1, reflecting the referral patterns in Reading where preschool screening is offered to all children between $3 \frac{1}{2}$ and 4 years. Forty-six per cent of subjects were male and $54 \%$ were female. Eighty (60\%) were hypermetropic (> +1.0 DS), 21 (16\%) were myopic $(<-0.5$ DS) and $70(52 \%)$ were astigmatic (> $\pm 1.0 \mathrm{DC})$. Thirty-nine (29\%) had a combination of errors and 47 (35\%) had anisometropia (> 1.0 DS). In 38 (29\% of total) the anisometropia was the main reason for the prescription. All children were prescribed full-time spectacles in an effort to improve a definite or strongly suspected defect of acuity, binocular vision or a squint. There were no pathologically high errors and 109 (82\%) were less than $\pm 3.00 \mathrm{DS}$ and / or $\pm 2.00 \mathrm{DC}$.

Mean initial improvement in visual acuity in either eye was $0.158 \operatorname{logMAR}$ units (range -0.3 to 0.82 ). This low figure reflects the large number of amblyopic children for whom dramatic initial improvement in acuity was unlikely with spectacles alone.

Only two children with accommodative squints had a noticeable cosmetic improvement in their deviation that might have favourably influenced compliance. In the other children the spectacles made only small cosmetic differences to the angle of the squint, but if they helped measures of binocularity this is included as a 'binocular benefit' below. For most children the most likely visual reasons for wearing spectacles were improvement in acuity or binocular vision (i.e. new or better binocular responses, stereoacuity or better control of an intermittent deviation). The six 'benefit' categories

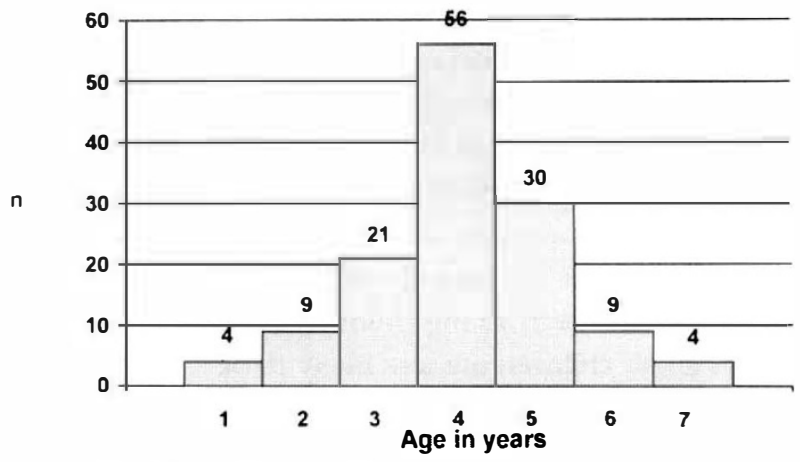

Fig. 1. Age distribution of subjects. 


\begin{tabular}{|c|c|c|c|c|}
\hline Scale rating & Definition & $\begin{array}{c}\text { No. in category } \\
\text { (\% of valid data) } \\
\text { when any VA } \\
\text { improvement counted } \\
\quad(n=120)\end{array}$ & $\begin{array}{l}\text { No. in category if } \\
>0.18 \text { log units used } \\
\text { as improvement } \\
\text { criterion }(\%)\end{array}$ & $\begin{array}{l}\text { Mean orthoptist } \\
\text { estimate of compliance } \\
\text { (out of maximum 100) }\end{array}$ \\
\hline Benefit 1 & Both VAs improved + BSV improved & $14(11.7)$ & $4 \quad(3.3)$ & 91.8 \\
\hline Benefit 2 & Both VAs improved, BSV same & $36(30)$ & $14(11.7)$ & 74.7 \\
\hline Benefit 3 & One VA improved, BSV improved & $18(15)$ & $16(13.3)$ & 86.2 \\
\hline Benefit 4 & BSV only improved & 11 (9.2) & $23(17.3)$ & 76.5 \\
\hline Benefit 5 & One VA only improved & $26(21.7)$ & $23(17.3)$ & 84.4 \\
\hline Benefit 6 & VA and BSV same/worse & $15(12.5)$ & $40(33.3)$ & 74.5 \\
\hline
\end{tabular}

Youngest children are excluded because accurate subjective VA was unobtainable.

$\mathrm{VA}$, visual acuity; BSV, binocular single vision.

(Table 3) were devised to differentiate those who obtained the most subjective benefit based on acuity, whether both-eyes-open vision improved, and whether binocular benefit was obtained. These categories focus on how much the child itself was likely to have benefited from or appreciated improvement in vision in the spectacles, not just on conventional ophthalmological measures (e.g. change in dissociated angle) that do not necessarily reflect the child's experience. The lower the number of the benefit category the more subjective improvement the child is likely to have had in everyday seeing conditions.

If more than one Snellen line ( $>0.18$ log units) is taken as the criterion, $50.6 \%$ of children gained little subjective improvement with their spectacles at first. Spectacles were frequently prescribed as the first stage in the treatment of unilateral or bilateral amblyopia and/or strabismus and immediate subjective improvement would not be expected.

Children generally liked their spectacles (mean score $75.5 / 100$ on the parental measure). There was a statistically marginal $(p=0.052)$ improvement in liking with age, with a small dip at 5 years. Liking of the spectacles did not differ significantly across the benefit categories, so those obtaining most subjective benefit liked their spectacles no better than those who obtained none.

Of those old enough to reply, $83(80.5 \%)$ of all the children said they could see better and only 20 (19.5\%) said they could not. There was an almost identical proportion of those who said they saw better or not in all categories of subjective improvement, i.e. even those not obtaining any measurable subjective improvement said they saw better.

Orthoptist and parental measures of overall compliance correlated very well $(r=0.653, p<0.000)$. Mean compliance was very good (orthoptist score 79.5, parental score 78.5 out of a possible 100) and both correlated very well with how much a child liked his or her spectacles (e.g. on parental measures $r=0.8083$, $p<0.000$ ). Age or sex had no effect on compliance, contrary to expectations, although the under-twos wore their spectacles the least (Fig. 2). There is also a consistent dip in compliance (also found for liking and friends' comments) at 5 years. Failure to find age effects may be due to the relatively small numbers of younger and older children.

Improvement in acuity did not correlate at all with general compliance ( $r=0.01, p=\mathrm{NS}$ ). When general compliance was analysed in relation to the benefit categories (Table 3 ) differences were generally not significant between those obtaining more or less benefit. The only significant difference $(p=0.033)$ between the individual groups was that group 1 (improvement in both visual acuities and binocular single vision (BSV)) wore their spectacles better than group 2 (both visual acuities better but no change in BSV), suggesting that improved BSV may be noticed by the children as well as bilateral improvement in visual acuity.

It therefore seems clear that subjective improvement in vision has little or no influence on the compliance of most children, and age has a doubtful effect except perhaps in the youngest group, so other factors were then considered.

The majority of spectacles fitted very well (mean score $76 / 100)$, but with significantly poorer fit $(50 / 100)$ in the under-twos. Regression analysis showed that fit was highly significantly related to compliance $(p<0.0000)$, with fit accounting for almost $20 \%$ of the total variance of compliance.

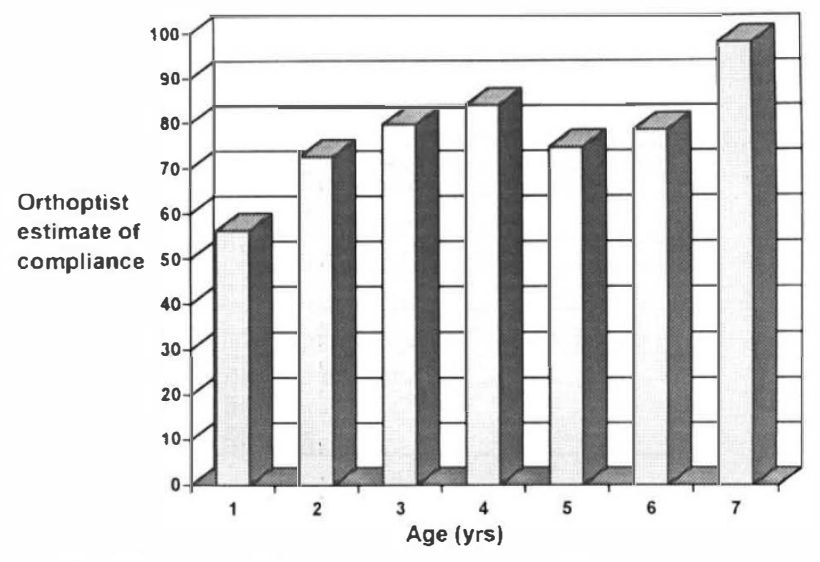

Fig. 2. Mean orthoptist estimate of overall compliance by age in years. 


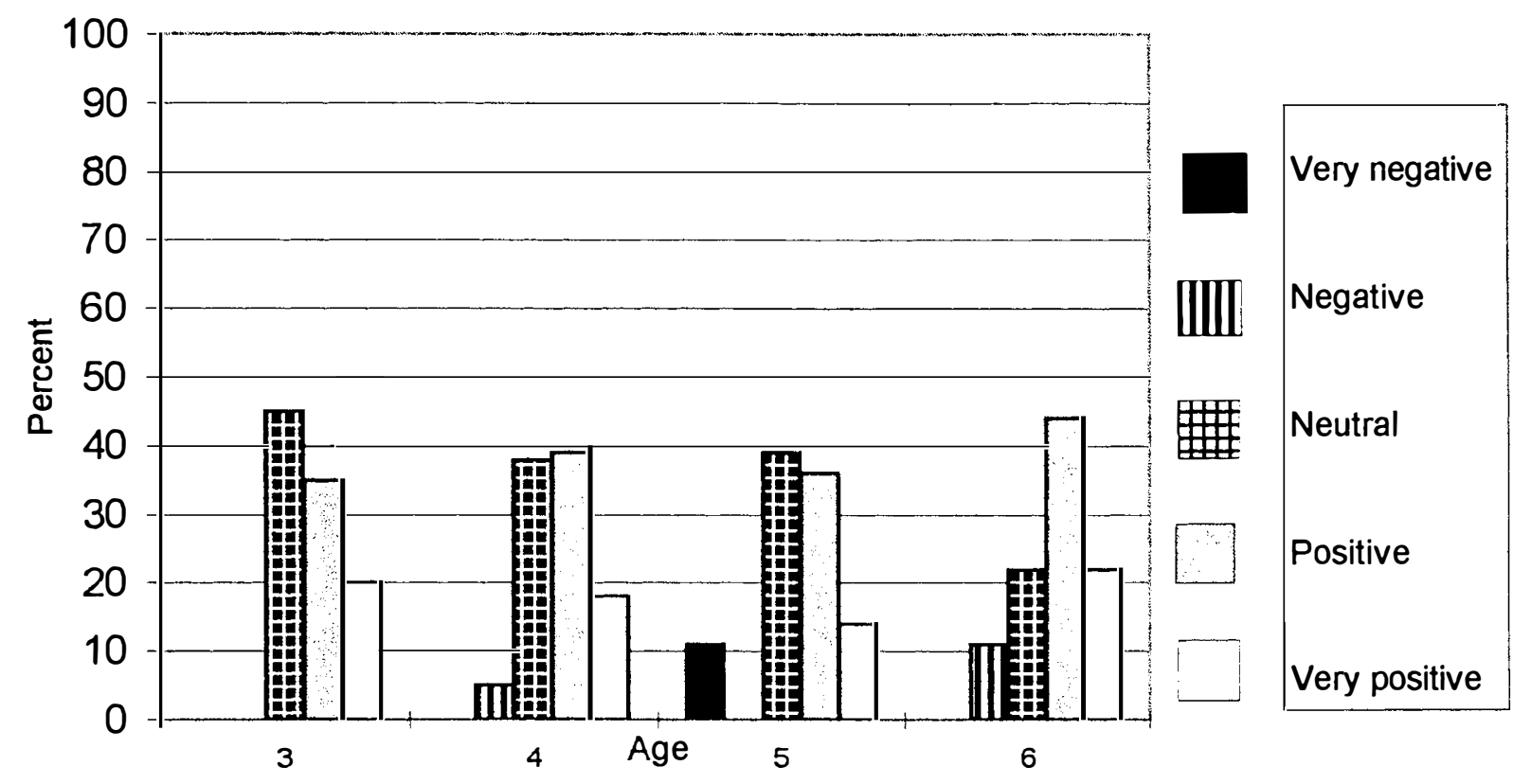

Fig. 3. Percentage of types of friends' comments by age.

Most parents found the experience of getting their children to wear spectacles much better than they had expected (mean score 75.1 when $0=$ much worse than expected and $100=$ much better than expected). The children (even the older ones) were rarely teased.

Friends' comments and those from people they did not like' (abbreviated to non-friends' in this paper) differed greatly. Both were coded on a five-point scale ( $1=$ very negative, 5 = very positive). Friends' comments were generally positive, and were highly significantly better than those of non-friends, which were generally negative $(t=8.95$, d.f. $=129, p<0.000)$. There were many neutral reactions, with the younger children usually receiving no comments at all, even from their friends. When asked about reactions of non-friends the younger children plainly had no idea what the question meant and either looked puzzled or made a comment such as But I like everybody'.

Figs. 3 and 4 illustrate the percentage of friends' and non-friends' comments in the age groups for which meaningful numbers were obtained. As the children get older the proportion of neutral comments reduces and,

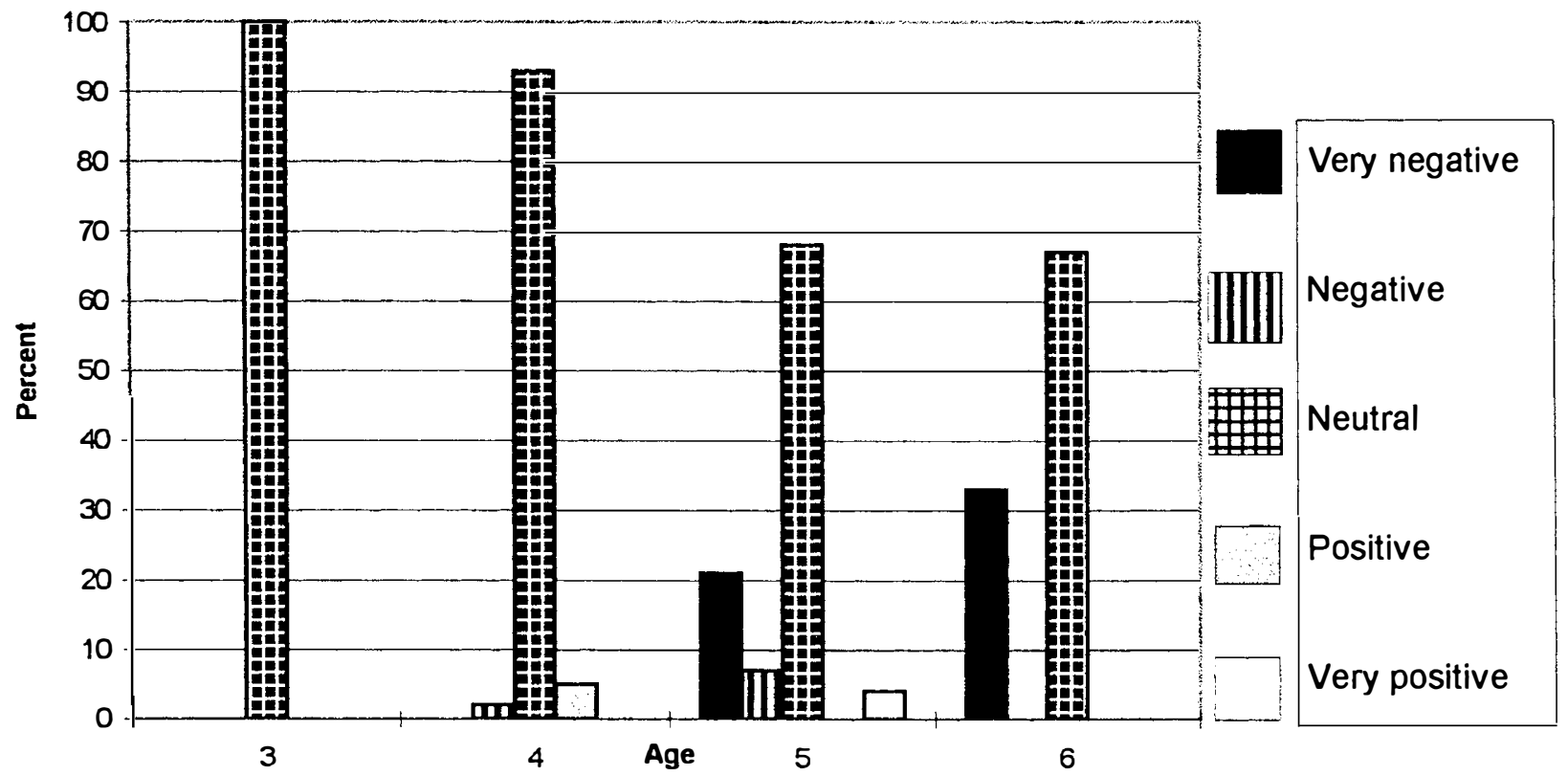

Fig. 4. Percentage of types of non-friends' comments by age. 
particularly in the case of non-friends, is replaced by negative comments (see Fig. 4). Of the 21 negative reports received across the age groups, $14(66 \%)$ came from nonfriends.

There was a highly significant trend $(F=3.36$, $p=0.003$ ) for better general wear the more favourable comments were received. Although not statistically significant (probably due to the very large number of neutral comments from younger children) there was a tendency for better wear to be associated with lack of adverse comments from non-friends.

If what other children think is a factor in how a child accepts spectacles, it is clearly the younger children who receive the most favourable comments and the fewest unfavourable ones. Looking at the actual comments written, the 4-year-olds in particular seem to be envied and admired for having spectacles, whereas by 5 and 6 years some spectacle-wearers are open to insults.

The overall incidence of adverse comments from both friends and non-friends was $1.7 \%$ for the under-fives, but $20 \%$ for the over-fives. If the neutral responses are disregarded the ratio of positive to negative comments is 23:2 for the under-fives but 24:17 for over-fives.

The parents generally received favourable comments about the spectacles, both in and out of the children's hearing (mean score $77 / 100$ ), but compliance was significantly lower $(t=2.08$, d.f. $=21.6, p=0.049)$ in those children whose parents received adverse comments. This suggests that not only the child's acquaintances but the parents' contacts may influence compliance indirectly.

Whether or not a parent wore spectacles now, or had done so as a child, did not influence compliance. However, if children over 4 years, who might be more aware of their parents' attitudes, were considered separately, having a parent who wore contact lenses was marginally associated with poorer compliance $(t=1.84$, d.f. $=18.97, p=0.08$ ). Further study using larger numbers might clarify whether this is a genuine effect.

\section{Discussion}

The data presented here clearly suggest that compliance with spectacles has little to do with visual improvement and much to do with the child's social world. It also supports the findings of McGraw, ${ }^{2,5}$ who suggests that spectacles are simply not salient to younger children in their reactions to others. The marginal influence of actual subjective visual improvement is fortunate for orthoptic patients who often obtain no subjective improvement at first. Good fit and approval from others seem the most influential factors in promoting good compliance. Although not studied here or elsewhere, clinical experience suggests that once spectacles are accepted they are rarely rejected until much older.

The only other database-listed references to the acceptance of spectacles are by Terry, ${ }^{1,6,7}$ who studied older children and found generally detrimental effects of spectacles on social judgments of others. The increase in positive comments from friends at 4 years and then of negative comments from non-friends at 5 years appears to reflect the increasing salience not only of others' appearance but also of spectacles in particular. Terry and Stockton ${ }^{6}$ suggest that level of cognitive development may determine the timing of the onset of salience and negative stereotypes of spectacle-wearers.

The optimum age for the child receiving maximum positive and minimum negative comments is 4 years. The dips in liking, compliance and positive friends' comments at 5 years, not maintained at 6 years, were interesting in combination and warrant further study. These were not individually significant and the data were not of sufficient quality to allow more sophisticated multivariate statistical techniques that might have demonstrated an effect. But 5 years of age is possibly a bad time to give children new spectacles, as they are making new social contacts at school where strangers may be more influenced by superficial appearance. Whether 6- and 7-year-olds genuinely like and wear their spectacles better (as suggested by Figs. 2 and 3), or have more problems with them as suggested by the social evidence, needs further study with larger numbers. However, this study demonstrates that older children are at higher risk of adverse comments, and that what other children say to a child influences whether that child wears his or her spectacles. Further research needs to concentrate on whether an old friend in new spectacles, a new acquaintance in spectacles, or an old friend in established spectacles is at least social disadvantage.

This evidence also has some implications for preschool screening. Studies of visual outcome comparing those screened at pre-school age and those not picked up until later have failed to prove a definite case for screening, and the controversy continues, but none have considered the child and family that are 'attached' to the eyes with defective vision. Totally aside from the wellknown arguments based on greater plasticity of a younger visual system, pre-school children settle easily into spectacles, receive fewer comments and are less vulnerable to negative peer pressure than older children. The evidence presented here suggests that spectacles are more happily accepted by a higher proportion of preschool children and their friends than they are by older children. They can progress effortlessly to full-time spectacle wear and occlusion without delays or unnecessary stress. Poor compliance with spectacles may mean wasted hospital visits, deferred occlusion and slower response to therapy. Hated spectacles are either not worn or can become the focus for family and school conflict, ultimately making ophthalmological treatment more protracted and stressful than necessary.

The author is most grateful to the ophthalmologists at the Royal Berkshire Hospital on whose patients this study was carried out, and to the orthoptists and patients in the Orthoptic Department who participated in the survey.

\section{References}

1. Terry RL. Social and personality effects of visual correctives. J Social Behav Personality 1990;5:683-95. 
2. McGraw KO, Durm MW, Durnam MR. The relative salience of sex, race, age, and glasses in children's social perception. J Genetic Psychol 1989;150:251-67.

3. Oppenheim AN. Questionnaire design, interviewing and attitude measurement. London: Pinter, 1992.

4. Bryman A, Cramer D. Quantitative data analysis for social scientists. London: Routledge, 1994.
5. McGraw KO, Durm MW, Patterson JN. Concept discrimination learning by pre-school children using facial stimuli differing in age, sex, race and eyeglasses.

J Gen Psychol 1983;108:193-202.

6. Terry RL, Stockton LA. Eyeglasses and children's schemata. J Social Psychol 1993;133:425-38.

7. Terry RL. Psychological considerations of visual correctives for children. Contact Lens Forum 1989;[March]:70-1. 